\section{ESTRUCTURA DE LA EMPATÍA: AUTOEXTRAÑAMIENTO E INTENCIONALIDAD INSTINTIVA}

\author{
Natalia Petrillo \\ Universidad e Buenos Aires/Wuppertal
}

\section{STRUCTURE OF THE EMPATHY: SELF ODDITY AND INSTINCTIVE INTENTIONALITY}

\begin{abstract}
The concept of "empathy" arises from the study of the constitution of an intermonadic community. Empathy takes the experience to be either not-originarily-now or not-mine. In both cases, I am extending my consciousness beyond the moment of being minenow to a type of re-presentation (Vergegenwärtigung). The notion of empathy is related to that of "temporality", insofar as Husserl claims that the empathy puts the other in a co-present. If each individual constitutes his/her own now, for his/her own consciousness, and if this level is distinctly separated from the level of intersubjective existence, then we cannot easily explain how the present is experienced as fundamentally the same for all subjects. In dealing with this difficulty, I will take up Husserl's considerations of temporalizing consciousness in the living present. The analysis will be completed with the drive-intentionality, which is very important in the constitution of the transcendental intermonadic community in a passive level.
\end{abstract}

KEY WORDS: Empathy, transcendental monadic community, driveintentionality, temporality.

En la quinta meditación de las Meditaciones cartesianas, dedicada al problema de la intersubjetividad, Husserl intenta explicar cómo es posible constituir el sentido de ser del otro sin violentar su alteridad. Es tarea de la filosofía trascendental resolver los problemas que atañen a la constitución del mundo desde una dimensión trascendental, esto es, desde mi ego trascendentalmente reducido. El problema del otro implica la justificación de su existencia en la actitud de la reducción, pues fuera de ella su existencia no es un legítimo tema de la fenomenología. La tarea es, entonces, explicitar el sentido "otro" y mostrar que él también experiencia el mismo mundo que nosotros'. La empatía, en tanto acto específico que puede dar cuenta de la experiencia de lo extraño, es un tipo especial de presentificación, mediante la cual el cuerpo físico extraño es aprehendido como manifestación de un cuerpo propio extraño. A diferencia de las otras presentificaciones, como el recuerdo, la fantasía, etc., lo característico del acto de empatía es poner la conciencia extraña empatizada en el modo de un "copresente"2. De
RESUMEN: A diferencia de las otras presentificaciones, lo característico del acto de la empatía consiste en poner al yo extraño en el modo de un "copresente". De este modo, el tratamiento de la estructura de la empatía está estrechamente ligado con la constitución tanto de la temporalidad propia como de la vida extraña. De allí que sea problemático determinar cómo es posible que, si cada sujeto tiene su propia temporalidad, no obstante surja un tiempo común.

PALABRAS CLAVE: Empatía, comunidad monádica trascendental, intencionalidad instintiva, temporalidad.

este modo, el tratamiento de la estructura de la empatía está estrechamente ligado con la constitución tanto de la temporalidad propia como de la extraña. Por lo que resulta problemático determinar cómo es posible que, si cada sujeto tiene su propia temporalidad, no obstante surja un tiempo común. En otros términos, se trata de elucidar cómo entran los otros en mi propia temporalidad ${ }^{3}$.

Si bien Husserl no ha dado una respuesta sistemática a estos interrogantes, algunos manuscritos tardíos indican el camino en que pueden ser respondidos. Con el propósito de explicar cómo la constitución de la intersubjetividad monádica se relaciona con la constitución de la propia temporalidad, se analizará, en primer lugar, la estructura de la empatía como autoextrañamiento (I). En segundo lugar, se examinará cómo es posible que el fluir pre-yoico se recubra con el fluir pre-yoico extraño (II). La última sección estará dedicada a esclarecer el fluir pre-yoico en relación con la intencionalidad instintiva universal (III). 


\section{Estructura de la empatía y AUtOEXTRAÑAmiento}

\section{Empatía: presentificación del ahora extraño}

La empatía pone un presente extraño, que tiene un tiempo determinado y propio. Husserl afirma que "mi presente viviente, mi 'presente' protomodal, contiene mi empatía, y en ésta presentifica al otro presente viviente" ${ }^{\prime \prime}$. La empatía -continúa Husserl- "pone lo dado en empatía como ahora y lo pone en el mismo ahora en que se pone a sí misma" ${ }^{\text {. }}$.

El ahora presentificado que pone la empatía no es un ahora rememorado, ello implica que este ahora no es puesto en la forma de un sí-mismo sino que más bien está puesto "con el ahora actual como él mismo"6. Se trata de un ahora en la presentificación que es puesto con el ahora actual como el mismo. La empatía posibilita, por tanto, una experiencia de una presentificación, sin presentificar, como en el caso del recuerdo, lo experienciado. Dicho de otro modo, el ahora empatizado es un ahora presentificado, pero no "visto" por sí mismo en el modo de la presentificación: "la simultaneidad de la empatía de lo empatizado no es 'una simultaneidad' que se observe a si misma"7. Lo peculiar de la empatía consiste entonces en poner un ahora extraño, es decir, un segunda conciencia original, que es irreductible a la primera.

Estas dos corrientes de conciencia no son, sin embargo, "dadas fenomenológicamente continuamente" ${ }^{\text {, }}$, es decir, el ahora de una no puede ser identificado sin más con el otro ahora, porque ambas temporalidades son inconmensurables una con otra y presentan corrientes de conciencia temporalizantes. En este sentido, el otro permanece siempre como un "presente no presente"

Ahora bien, ambas corrientes de conciencia no se asemejan en sus contenidos, sino más bien en su estructura. Gracias a la empatía se constituye un co-presente de lo extraño como una modificación de la auto-experiencia egológica. En tanto el cuerpo físico extraño es aprehendido como cuerpo propio, esta aprehensión analógica motiva la presentificación de una primordialidad extraña como modificación de la mía propia ${ }^{10}$.

De este modo, la experiencia corporal extraña es caracterizada como tal, siempre y cuando tenga lugar una modificación de mi presente en una presentificación ${ }^{11}$. La iden- tificación de ambos ahoras está mediada por la relación de ambos yoes en relación con el tiempo objetivo del cuerpo propio. Dicha relación entre el cuerpo físico extraño y mi cuerpo propio primordialmente constituido presupone una sintesis del presente original con el presente presentificado en el modo de la empatia ${ }^{12}$. En este sentido, Fink caracteriza también la empatía como formación de unidades sintéticas de fases de proto-temporalización actual ${ }^{13}$. Dado que la reducción trascendental no nos da otra subjetividad trascendental más que la nuestra, la constitución del otro parte necesariamente de nuestro presente viviente ${ }^{14}$.

\section{Empatía y autoextrañamiento}

Para poder explicitar el proceso de proto-temporalidad, se pueden diferenciar tres estadios de temporalización: la estructura yoica, el fluir sin yo y el fluir pre-yoico.

El análisis estructural del presente primitivo (el fluir permanentemente viviente) nos conduce a la estructura yoica (Ichstruktur) y a la constante capa inferior, que la funda del fluir sin yo (ichlöses Strömen), que, por medio de una pregunta retrospectiva consecuente por lo que también hace posible a y presupone la actividad sedimentada, retrotrae a lo radicalmente pre-yoico (das radikal Vor-Ichliche) ${ }^{15}$.

El primer nivel de temporalización del presente viviente conduce a un último yo operante como lugar originario de toda constitución. Se trata del fluir de los actos del yo como polo de acción y afección.

Problemáticos son los otros dos niveles de temporalización, el fluir sin yo y el fluir radicalmente pre-yoico. Eugen Fink sostiene que se trata de una proto-vida que antecede a la distinción ego y alter ego. Según tal interpretación habría una proto-vida, que no es ni una ni muchas, ni fáctica ni esencial. Esta proto-vida que se pluraliza a sí misma, sería lo último que opera absolutamente, por tanto, se encontraría en un nivel pre-egológico previo a toda individuación y pluralización ${ }^{16}$.

Por el contrario, D. Zahavi refuta dicha interpretación y sostiene que el fluir sin yo es una mera abstracción dentro de la subjetividad constituyente. Zahavi intenta esclarecer la distinción entre "fluir sin yo" y "fluir pre-yoico" del siguiente modo. En el caso del "fluir sin yo", la expresión "sin yo" no se refiere a la falta de un yo acompañante, sino 
"a la proto-pasividad del fluir, que le es sustraida al flujo yoico"17. Pero cuando Husserl habla de un nivel pre-yoico, no se refiere a un fundamento previo a la individuación, sino a un nivel, que tiene que ver con la constitución del yo como objeto temático de reflexión. Puede entenderse entonces al segundo nivel como un fluir carente de yo. En un estrato más profundo, este fluir sin yo conduce a un fluir pre-yoico, es decir, a un yo anterior al desarrollo de habitualidades instituidas por el comportamiento yoico. Este protonivel de constitución temporal se encuentra antes de toda constitución aperceptiva, y desde el punto de vista egológico, se refiere a una proto-kinestesia, que se dirige a una proto-hyle sin la participación del yo y de un modo indiferenciado ${ }^{18}$.

Ahora bien, la tematización del fluir pre-yoico se relaciona con el problema de la empatía, ya que este fluir tiene cierta relación con el presente viviente de los otros. La auto-temporalización del ego en el pre-presente originario abre una infinitud de egos originarios, que se implican mutuamente. En un manuscrito de 1934-1935, Husserl explica que mi presente originario lleva en sí primordialidades extrañas, apresentadas. El yo originario tiene en sí un ser originario puesto como ego, que implica un ser empatizado. En este sentido, Husserl afirma que el otro está constituido en mí como "subjetividad co-presente fluyente" ${ }^{19}$.

De alli que, dado que el presente primigenio presentifica en este tercer nivel de temporalización un presente viviente extraño, es posible hablar de una "escisión" (Spaltung) del tiempo en un presente original propio y en un presente extraño presentificado. Husserl habla de una "auto-multiplicación" (Selbstvervielfältigung) del ego primordial y también de un "auto-extrañamiento" (Selbstentfremdung): "el resultado de la empatía como efectuación es una modificación de presentificación de la espera primordial fundante como 'otra' primordialidad, como primordialidad 'extraña'"20, que a su vez implica un "autoextrañamiento del yo primordial y de su esfera primordial" ${ }^{21}$. Con ello se escinde en el autoextrañamiento del yo primordial lo proto-modal del yo extraño. Así la proto-temporalización del presente viviente es reconducida a mi temporalización egológicamente reducida y luego a partir de ahí a la temporalización intersubjetiva ${ }^{22}$.

Con lo dicho, puede concluirse que la auto-temporalización posibilita una nueva temporalidad en la forma de coexistencia entre egos primigenios. Del fluir pre-yoico surge primero una coexistencia entre presentes comunes: "una multiplicidad abierta de otros y siempre otros en la unidad de una coexistencia originaria, esto es, presente"23. Si bien Husserl habla explícitamente de coexistencia, para subrayar la fuente última de la temporalización intersubjetiva, se refiere a una "proto-coexistencia". En el presente viviente propio experiencio el presente extraño protomodal y "con ello la proto-coexistencia de mi ser propio y del ser extraño"24.

En el horizonte del auto-extrañamiento se constituyen mi mónada protomodal y las otras mónadas en una coexistencia universal, en un tiempo monádico total. Así, el autoextrañamiento de la empatía remite a una síntesis entre mi presente original y el co-presente presentificado en la forma de una proto-coexistencia, "donde el tiempo del uno se recubre con el del otro"25. Tal recubrimiento presupone un tiempo "sobre-monádico o intermonádico de nivel superior" ${ }^{126}$, es decir, se trata de un fluir uno-en-otro continuo de los tiempos monádicos. En lo que sigue se intentará explicitar cómo se recubren ambos fluires pre-yoicos.

\section{EL TODO MONÁdICO INTERNAMENTE UNIFICADO}

Con el objetivo de explicar cómo ambos fluires pre-yoicos se recubren, se hará primero una breve referencia a la doble intencionalidad de la retención.

Husserl distingue entre la retención de las fases de los objetos inmanentes a través de una intencionalidad transversal y la retención de las fases del curso absoluto a través de una intencionalidad longitudinal. En virtud de la doble intencionalidad de la retención, no sólo captamos fases pasadas de objetos inmanentes en nuestro curso de conciencia, sino también las fases transcurridas de nuestra conciencia primaria de ellas. No se trata de dos intencionalidades separadas unas de otras, sino de dos modos de descripción de uno y el mismo fenómeno. La intencionalidad longitudinal se dirige a la unidad del curso absoluto y constituye la proto-forma retención-protención. La intencionalidad longitudinal funciona como base para la intencionalidad transversal, que se dirige a fases pasadas y gracias a la cual las fases retenidas del objeto inmanente pueden ser captadas ${ }^{27}$.

ARBOR CLXXXV 736 marzo-abril [2009] 369-377 ISSN: 0210-1963

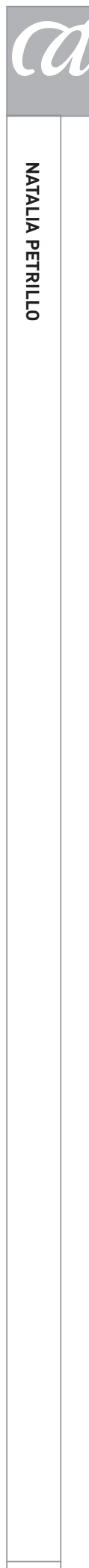


Si bien Husserl no utiliza los conceptos de "intencionalidad transversal" e "intencionalidad longitudinal" para referirse a la empatía, puede hablarse -tal como propone R. Walton- de una doble intencionalidad de la apresentación en relación con la efectuación de la empatía ${ }^{28}$.

Teniendo en cuenta una retención que no está dirigida a las fases pasadas de nuestros actos sino al propio curso, puede suponerse que Husserl esboza la idea de una apresentación que no está dirigida mediatamente, a través del cuerpo ajeno, a los actos de otro yo sino inmediatamente a las fases de un curso intersubjetivo. Esto lleva a distinguir dos dimensiones dentro de la apresentación del otro yo admitiendo una análoga doble intencionalidad.

Por un lado, la apresentación del otro en el sentido habitual tendría su condición de posibilidad en una apresentación primigenia que da cuenta de la unidad entre cursos de conciencia en la misma forma en que la intencionalidad longitudinal da cuenta de la unidad dentro de cada curso absoluto. Tal apresentación no se sustenta en la aprehensión analógica del otro cuerpo, sino en una red de implicaciones inherentes al presente viviente. Con otras palabras, su base motivacional no es la semejanza que conecta al cuerpo que está allí con mi cuerpo propio, sino que el co-presente remite al presente viviente del otro ${ }^{29}$. Del mismo modo que mi pasado presupone mi presente viviente, presupone la apresentación del co-presente extraño también un presente viviente extraño. Husserl sostiene: "el ser implicado de mi pasado en el presente fluyente, el ser implicado de otras mónadas en mi mónada, en el presente fluyente: la implicación como trascendentalmente simultánea" ${ }^{30}$.

Por otro lado, la intencionalidad transversal se dirige a una fase de la temporalidad inmanente, de modo tal que la intención se dirige a cada fase del horizonte de una totalidad monádica. Del mismo modo que el ahora implica un horizonte de proto impresión, retención y protención, pertenece también al horizonte un co-presente presentificado, que en sí mismo puede mostrar modificaciones intencionales.

Así como se une a un horizonte viviente de retenciones y protenciones que es previo a las presentificaciones, el presente viviente se enlaza mediante una apresentación originaria a una "comunidad arcóntica" ${ }^{31}$. La siguiente cita puede entenderse a la luz de la intencionalidad transversal: "el 'presente' permanente y primigenio de la totalidad monádica interioramente unificada, en la que está constituido todo tiempo y todo mundo, monádico y mundano"32. Al horizonte de este co-presente pertenece "un campo de 'protención' en sentido amplio"33. K. Held agrega que el co-presente presentificado remite a un horizonte abierto infinito, que conduce a la totalidad de todos los yoes trascendentales como a sus auto-temporalizaciones. K. Held concluye que "en el co-presente está consciente concomitantemente en el modo del horizonte el 'todo monádico'"134. En este sentido, habla Husserl de un "presente primigeniamente permanente del todo monádico internamente unificado" ${ }^{35}$. La presuposición de una intencionalidad longitudinal permite entender la siguiente afirmación de Husserl: "El otro es para mí igualmente, pero su para-sí es a la vez un para-mí en la forma de mi potencialidad de la apresentación; pero él mismo es apresentado en mí y yo en él; yo llevo todos los otros en mí como otros apresentados o que han de ser apresentados en sí mismos y como llevando en sí igualmente a mí mismo"36.

Nos encontramos en una relación con el otro que depende de una intencionalidad que remite a los otros cursos de conciencia antes de toda experiencia concreta del otro. Si bien en este punto del análisis la referencia a otros momentos de un curso intersubjetivo permanece abstracta, encuentra su cumplimiento, en un segundo paso, a saber, en el análisis genético de un impulso universal que pasa por todas las subjetividades. Se trata de una proto-intersubjetividad instintiva sustentada en una protointencionalidad instintiva de comunicación, que será analizada a continuación.

\section{INTENCIONALIDAD INSTINTIVA UNIVERSAL}

Al referirse al "fluir continuo del uno-en-otro en del tiempo monádicamente propio" ${ }^{37}$, Husserl plantea la pregunta acerca de la presuposición de una intencionalidad instintiva universal.

En la sección anterior, se ha intentado mostrar, siguiendo el planteo de R. Walton, que el presente primigenio propio implica una vida trascendental extraña. El punto de partida de los análisis de Husserl en relación con la intencionalidad 
instintiva es la proto-afección que está dirigida a impulsos extraños: "la nueva afección, el nuevo instinto, que se dirige en general a los co-sujetos y a los sujetos"38. Previo a la distinción sujeto-objeto, el impulso busca y encuentra una afección extraña. Se trata de impulsos extraños que afectan al fluir pre-yoico, despertando nuevos intereses. Surge así el interés nuevo en el otro, y en verdad -agrega Husserl- "en su parificación con mi propio yo" ${ }^{39}$.

Por un lado, dado que el yo no se afecta todavía a sí mismo, pues, repitámoslo una vez más, nos encontramos en un nivel instintivo de análisis, en el cual no se ha formado aún una conciencia constituyente, no es posible que el estímulo de la proto-hyle provenga de mí mismo. Por el otro, aquello que se constituye en mi presente viviente es parificado sin corporalidad externa. Por ello, no es este interés todavía interés en el cuerpo propio externo. Puede afirmarse entonces que la proto-hyle, esto es, lo primero extraño al yo, proviene de los otros pre-yoes, cuyas intencionalidades forman una conexión originaria e instintiva ${ }^{40}$. Esta relación instintiva entre lo empatizante y lo empatizado está predada y opera como condición de posibilidad para la empatía en un nivel superior. Por tanto, la empatía no es sólo posible gracias a una estructura de experiencia común entre lo empatizante y lo empatizado, sino más bien gracias a una relación instintiva.

Husserl se refiere a un impulso intersubjetivo, en el que yace "la relación al otro como otro y a su impulso correlativo" ${ }^{41}$. Un enlace instintivo configura una proto-intersubjetividad: "proto-intencionalidad instintiva de la comunalización"42. Esta proto-intencionalidad instintiva de la comunalización es una pre-conciencia de comunidad. No se trata de una intencionalidad que está dirigida al otro en la forma de la empatía, puesto que su meta es alcanzada por los pre-yoes inmediatamente en el modo de la presentación mediante la unidad de ambas esferas primordiales. Con otras palabras, la intención instintiva encuentra su meta sin la mediación de una presentificación porque desde el principio el protoinstinto está relacionado inmediatamente con el otro: "En la simple satisfacción protomodal no tenemos dos satisfacciones que deben separarse en cada caso en una y otra primordialidad, sino una unidad de ambas primordialidades que se establece por el uno-en-otro de las satisfacciones" ${ }^{\prime \prime 3}$. Con ello, se plenifican dos proto-intencionalidades vacías en el misma experiencia, sin que estén referidas a un objeto, sino a un instinto semejante en el otro.
De ello se sigue que la intencionalidad instintiva está en estrecha relación con la problemática de la empatía, pues en cada presente primordial hay instintos de nivel superior que trascienden en el otro presente y se relacionan unos con otros. Los instintos están de este modo implicados unos "en" otros intencionalmente ${ }^{44}$. Pero esta experiencia, en la que dos proto-intencionalidades vacías se plenifican, tiene lugar en un pre-tiempo intersubjetivo, de manera tal que el todo monádico internamente unificado implica una "temporalización intersubjetiva en el pre-tiempo intersubjetivo"45. Con la expresión "pre-tiempo", Husserl parece referirse al horizonte último y oscuro de la intencionalidad instintiva: "En el pre-tiempo como tendencia de desarrollo oscura, como intencionalidad instintiva, lo último en el despertar del verdadero humanismo"46.

Con esta caracterización de la intencionalidad instintiva en relación con la proto-hyle es posible complementar la distinción propuesta por R. Walton entre intencionalidad transversal y longitudinal en el caso de la apresentación de la empatía.

Dado que esta relación originaria en el modo del sentimiento del pre-yo a lo extraño al yo es inseparable de los instintos no desvelados, resulta que en el caso del sentimiento en el fluir originario no se trata de un objeto, sino de una unidad de sentimiento total, que corresponde a la proto-hyle indistinta como proto-forma del mundo ${ }^{47}$. Como estar-ahí siempre sintiente del yo, el impulso dirigido al otro unifica lo proto-hylético en la corriente primigenia de vida, de modo que la totalidad de la proto-hyle es constantemente afectada (intencionalidad longitudinal).

Pero en la fase del comienzo de la génesis trascendental no se trata de una única intención instintiva, sino de muchas intenciones instintivas que se afectan mutuamente, de modo que el horizonte instintivo del pre-yo no permanece sin relación con otros. También dentro de esta unidad de afección hay "afecciones especiales [tanto] en 'lucha' unos-con-otros, [como] en lucha por el yo"48. Dicho de otro modo, la unidad de la proto-afección no es otra cosa que un instinto total para diversos instintos especiales. En este punto, hay que agregar que las afecciones especiales hyléticas de cada afección total, que ya ha transcurrido, pueden ser afectadas transversalmente (intencionalidad transversal).

ARBOR CLXXXV 736 marzo-abril [2009] 369-377 ISSN: 0210-1963

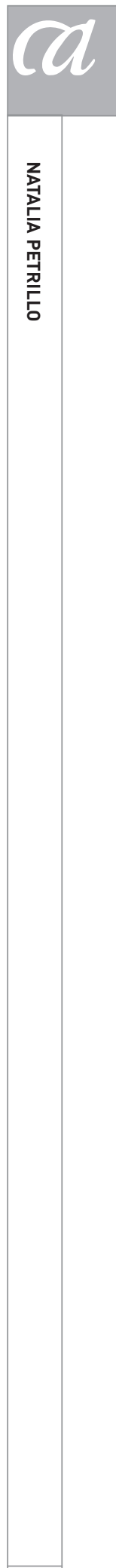

373 
Importa destacar que la unidad, que se constituye como dato hylético, es al mismo tiempo, unidad de un sentimiento, que lleva consigo la caracteristica del sentimiento. Así como la intencionalidad longitudinal en el caso de la intencionalidad de la retención está dirigida a las fases del curso absoluto, en el caso de la intencionalidad instintiva se dirige la intencionalidad longitudinal a un instinto total, "que abarca todos los instintos especiales y que opera a través de sus revelaciones especiales, es decir, a través de sus constituciones especiales" ${ }^{19}$. La intencionalidad transversal está dirigida al instinto especial, es decir, a cada fase del instinto total.

El instinto total opera en cada ego particular como un impulso originario. Además, el instinto total pasa por los instintos sociales y generativos en el alter ego. Esta continuidad intersubjetiva, que es producida por la operación de las intenciones instintivas de las mónadas singulares, no es caótica, sino un todo estructurado dirigido a la constitución de un mundo intersubjetivo ${ }^{50}$. Este horizonte instintivo de horizonte superior es el mundo del pre-yo que implica un co-mundo. Con ello, queda ejecutada la explicitación de los instintos no-desvelados, esto es, del pre-yo.

Aquí surge el problema de la empatía como motivo para la tematización del pre-yo. La vida del otro puede ser entendida sólo como secundaria frente a la "proto-vida de una incorporación de lo subjetivo"51. El crecimiento está dirigido en parte a sí mismo, en parte a los otros: "Con ello la constitución del mundo entero parece ya 'instintivamente' predelineada para mi" ${ }^{52}$. Esto significa que el tender hacia el mundo objetivo implica en el pre-yo una intención instintiva teleológica. Mediante un nuevo despertar de pre-yoes, Husserl elabora la concepción de una comunidad instintiva constituida, a partir de la cual el mundo se constituye como horizonte.

Cabe notar que la tendencia universal que pasa a través de todas las mónadas trascendentales puede desarrollarse. Esta teleología universal abarca todos los instintos posibles trascendentales como una teología universal de las mónadas singulares. Es posible dar un paso más y afirmar con Husserl que la intencionalidad instintiva universal ha de ser entendida como teleología universal, en el que se fusionan todo los instintos trascendentales de las mónadas singulares como teleologías especiales: "Instinto transcen- dental -en un sentido la tendencia universal que atraviesa mediante la totalidad de la intencionalidad del ego- la teleología universal constante" ${ }^{n 3}$.

\section{Conclusión}

Se ha intentado mostrar que la efectuación de la empatía consiste en el auto-extrañamiento del propio presente primigenio. Cuando la empatía pone un co-presente extraño, que por principio no puede ser visto por sí mismo, pero que coexiste con el mío, surge un auto-extrañamiento dentro de la esfera primordial, que al mismo tiempo es un autoextrañamiento de la temporalización extraña. Con ello, en la propia temporalidad coinciden dos presentes intencionales primigenios. La explicitación de la experiencia de lo extraño lleva a la explicitación del co-presente del otro como proto-modo de la experiencia de lo extraño $0^{54}$.

Para explicar cómo esta proto-coexistencia de mi fluir pre-yoico se recubre con el co-presente presentificado del otro, se ha seguido la lectura de R. Walton, según la cual hay que tener en cuenta la doble intencionalidad de la apresentación en el caso de la empatía. Para ello, hemos brevemente recordado que Husserl hace una distinción entre la intencionalidad longitudinal y transversal en el caso de la retención. La intencionalidad longitudinal como proto-forma de la apercepción tendría la función de unificar ambos fluires primigenios. Ello es posible porque el co-presente extraño presentificado implica también un presente extraño viviente. La presuposición de una intencionalidad transversal orientada a cada fase de la temporalidad co-presentada posibilitaría que la intención que se dirige a cada fase del horizonte, pueda apresentar transversalmente las fases pasadas del horizonte. De este modo, el horizonte de una temporalidad de vida extraña puede ser empatizado transversalmente.

De allí resulta el momento más decisivo de la efectuación de la empatía: la relación entre ambos cursos de conciencia como "copresencia de primordialidades" (Primordialitätenkompräsenz) ${ }^{55}$. Este "co" del co-presente es el modo de una "protocomunalización" ${ }^{56}$, en la que yo co-vivo en mi vida primordial a la vez con la vida del otro en el modo de la empatía. Husserl habla de una proto-coexistencia entre mi corriente originaria pre-yoica y la del otro. De allí que 
se produzca una "unidad de vida" y una "unicidad-yo-tú" 57. Con el fluir pre-yoico se muestra que el presente protomodal se constituye en diversos momentos no-yoicos.

Según Iso Kern, el otro "como co-sujeto y como co-presente fluyente es inseparable del presente fluyente del ego"58. Esta relación de dependencia mutua consiste en una necesareidad trascendental de la coexistencia, a saber, del "ser-uno en la co-vida" (Einsseins im Mitleben) ${ }^{59}$, de modo que "el tiempo monádico, el tiempo todo de las mónadas sea enraizado en mi presente primigenio [...]"60. Esta necesariedad trascendental del ser-junto-con se expresa también como "proto-hecho metafísico"61 en la forma de un ser-uno-en-el-otro intencional de lo absoluto.

Cabe destacar que sólo se ha tematizado el nivel inferior de la empatía. Husserl diferencia la empatía auténtica de la inauténtica. Mientras que la primera se dirige al yo y a sus acciones y posiciones, nuestro análisis se ha limitado sólo a la explicitación del nivel de la autoconstitución pasiva con sus horizontes instintivos abiertos. En este sentido, Husserl habla no sólo de la efectuación de la empatía, sino de la "proto-empatía" 62 para designar la constitución del presente primigenio intersubjetivo.

La tercera parte de este trabajo ha intentado completar y concretizar el carácter abstracto de la doble intencionalidad en el caso de la empatía mediante el análisis de la intencionalidad instintiva.

En dicho contexto, hemos visto que la proto-hyle tiene un rol importante para la autoconstitución del ego y del alter ego, en tanto opera antes de la diferenciación yo-yo extraño. La proto-hyle es entonces entendida como la célula originaria de la alteridad y también como el núcleo protomodal que es inseparable de mí en el proceso de formación continua de sentido ${ }^{63}$. Las "ventanas" de las mónadas no han de ser buscadas por tanto "arriba" en la apercepción o en la transferencia analógica, sino en el instinto originario, que ya siempre está dirigido al otro y encuentra alli su plenificación.

Queda por remarcar que los instintos presuponen una cierta autoafección. En ellos reposa la constitución de la intersubjetividad a partir de una pasividad última. Según R. Kühn, tanto la pasividad radical como la intersubjetividad radical son igualmente originarias, sin que se pueda indicar o presumir una mediación ${ }^{64}$. De este modo, la subjetividad absoluta es entendida como afección de vida, a partir de la cual los impulsos intersubjetivos se forman como primera expresión o exteriorización de la autoexplicación trascendental.

Estos análisis nos llevan a plantear si en última instancia el yo puede ser separado del no-yo. Nos encontramos con ello en la esfera de la pasividad originaria, de donde surge la separación de lo hylético y lo noético, es decir, en la esfera del proto-ser absoluto, a partir de la cual se diferencia el yo del no-yo65. Es posible afirmar -junto con Waldenfels- que lo extraño comienza en nosotros mismos y no fuera de nosotros. La "proto-distinción" (Urscheidung) entre lo propio y lo extraño en el proceso de diferenciación presupone una cierta indiferencia ${ }^{66}$. El ser-uno-en-otro intencional comprende, por tanto, un recubrimiento o fusión de lo propio y lo extraño. En este sentido, la monadología puede entenderse como el reconocimiento de la co-originariedad del otro, cuya aparición no ha de ser pensada como objeto que nos limita desde fuera, sino precisamente como una unidad en la dualidad y como una dualidad en la unidad del ser-con en la proto-actualidad del presente y de la co-actualidad del co-presente ${ }^{67}$.

\section{NOTAS}

1 Hua I, 124. Su importancia radica en que la teoría de la empatía también "co-fundamenta una teoría trascendental del mundo objetivo" (Hua I, 124). La sigla corresponde

Recibido: 10 de diciembre de 2007 Aceptado: 5 de enero de 2008 a Gesammelte Werke-Husserliana, vols. I-XXXVIII, Dordrecht/Boston/
London, Kluwer Academic Publishers (con anterioridad: Den Haag, Martinus Nijhoff), 1950-2006. Se indica a continuación el volumen y la página. Se sigue la traducción española de la quinta Meditación de García-Baró en Husserl, Edmund, Meditaciones cartesianas, México, FCE, 1996, p. 152. De aquí en adelante: $M C$. 
2 Hua VIII, 134.

3 Cf. Hua XV, 353. Cf. Ludwig Landgrebe, Faktizität und Individuation, Hamburg, Meiner, 1982, p. 51.

4 Hua XV, 331. En otros textos afirma Husserl: "La esfera primordial extraña está en recubrimiento sintético con la mía" (Hua XV, 634). "El otro está en mí presentificado. Yo [...] tengo su presente como copresente." (Hua Mat VIII, 56). Cf. Hua Mat VIII, 61, 91, 365.

5 Hua XIII, 189; Husserl, Problemas fundamentales de la fenomenología, Madrid, Alianza, 1994, p. 125. De aquí en adelante PF. Husserl reitera lo dicho anteriormente y nos dice que "el acto de empatía y el acto empatizado pertenecen al mismo tiempo. La empatía pone lo empatizado como ahora, y lo pone como el mismo ahora en que se pone a sí misma" (Hua $\mathrm{XIII}, 227 ; P F$, p. 193).

6 Hua XIII, 227; PF, p. 193.

7 Hua XIII, 189. Cf. Hua XIII, 221. Cf. Hua Mat VIII, 57.

8 Hua XIII, 222.

9 Hua XIII, 311.

10 Cf. Hua XV, 588, 642.

11 Cf. Römpp, Georg, Husserls Phänomenologie der Intersubjektivität und ihre Bedeutung für eine Theorie intersubjektiver Objektivität und die Konzeption einer phänomenologischen Philosophie, Phaenomenologica 123, Dordrecht/Boston/London, Kluwer Academic Publishers, 1991, p. 102.

12 Cf. Hua XIII, 227. Cf. Hua XV, 642.

13 Cf. Van Kerckhoven, Guy, Mundanisierung und Individuation bei $E$. Husserl und E. Fink. Die VI. Cartesianische Meditation und ihr "Einsatz", Orbis Phaenomenologicus, Studien 2, Würzburg, Könighausen \& Neumann, 2003, p. 298.

14 Cf. Hua Mat VIII, 105.

15 Hua XV, 598.
16 Cf. Zahavi, Dan, Husserl und die transzendentale Intersubjektivität. Eine Antwort auf die sprachpragmatische Kritik, Phaenomenologica 135, Dordrecht/Boston/London, 1996, p. 56. Otra interpretación proporciona L. Landgrebe. Su tesis consiste en que el proto-fluir está más allá de lo interno y lo externo, de lo subjetivo y lo objetivo, de lo espiritual y de lo material, de la forma y la materia (cf. Landgrebe, Faktizität und Individuation, p. 85). El autor entiende la subjetividad trascendental como proceso creativo. No discutiremos aquí esta tesis.

17 lbidem.

18 Cf. Hua Mat VIII, 328.

19 Hua Mat VIII, 56. Cf. Hua XV, 586, $587,596,605,667$.

20 Hua XV, 634.

21 Hua XV, 634. Cf. Hua XV, 635, 639640.

22 Cf. Hua Mat VIII, 85.

23 Hua XV, 335. "Las mónadas juntas tienen una temporalidad intermonádica, una forma de coexistencia, [...] una forma de coexistencia subjetivatrascendental" (Hua Mat VIII, 173). Cf. Hua XIV, 360.

24 Hua XV, 334. El subrayado es nuestro. Cf. Hua Mat VIII, 135.

25 Hua XV, 331-332. "Pero ambos tiempos, en tanto la coexistencia alcanza, se 'recubren', y eso es precisamente, nosotros existimos en el modo de un coexistir continuo en el presente" (Hua XV, 372). Cf. Hua XV, 637.

26 Hua $X V$, 343. Cf. Hua XV, 332, 334335.

27 Cf. Hua X, 81-82, 379.

28 Cf. R. Walton, reseña César Moreno Márquez, La intención comunicativa: Ontología e Intersubjetividad en la fenomenología de Husserl, Sevilla, Thémata, 1989, en Husserl Studies, vol. 10, n. 2 , 1993-1994, p. 145.
29 Cf. Idem, p. 146.

30 Hua Mat VIII, 22. Cf. Hua Mat VIII, 57, 58. Cf. Hua XV, 336.

31 Hua XV, 670.

32 Hua XV, 666.

33 Hua XV, 94.

34 Held, Klaus, Lebendige Gegenwart. Die Frage nach der Seinsweise des transzendentalen Ich bei Edmund Husserl, entwickelt am Leitfaden der Zeitproblematik, Phaenomenologica 23, Martinus Nijhoff/Den Haag, 1966, p. 158. Cf. Hua Mat VIII, 278.

35 Hua XV, 666.

36 Hua Mat VIII, 56. Cf. Hua XV, 371; Hua Mat VIII, 317.

37 Hua XV, 335. Cf. Hua XV, 367-368. Cf. Hua Mat VIII, 58.

38 Hua Mat VIII, 338. Husserl sostiene que "ya la pasividad, la vida instintiva puede producir una conexión intersubjetiva" (Hua XIV, 405).

39 Hua Mat VIII, 339.

40 Cf. Hua Mat VIII, 100, 183, 339. Cf. Hua XV, 582.

41 Hua XV, 594.

42 Ms. E III 10, 8b. citado por Landgrebe, "Die Phänomenologie als tranzsendentale Theorie der Geschichte", in Phänomenologische Forschung, Band 3, Freiburg/München, Karl Alber, 1976, p. 41.

43 Hua XV, 594. Cf. Walton, "Instintos, generatividad y tensión en la fenomenología de Husserl", en Naturaleza Humana. Revista Internacional de Filosofia e Prácticas psicoterápicas, vol. 4, n. ${ }^{\circ}$, San Pablo, 2002, pp. 253-292.

44 Cf. Hua XV, 595.

45 Hua XV, 597.

46 Ms. E III 1, 4b. Los manuscritos se citan según las transcripciones existentes en el Archivo Husserl. Agradezco al Prof. Dr. Ullrich Melle, Director del Archivo Husserl de Lovaina 
(Katholieke Universiteit Leuven), la autorización para citar manuscritos inéditos de Edmund Husserl.

47 Cf. Nam-In Lee, Edmund Husserls Phänomenologie der Instinkte, Phaenomenologica 128, Dordrecht/Boston/London, Kluwer Academic Publishers, 1993, p. 121.

48 Ms. E III 9, 16 b.

49 Ms. E III 9, 18a.

50 Cf. Nam-In Lee, op. cit., p. 226.

51 Hua VIII, 63. Cf. Hua VI, 189.

52 Hua XV, 385. Cf. Hua Mat VIII, 170.

53 Hua Mat VIII, 260.

54 Cf. Fink, Eugen, VI. Cartesianische Meditation. Teil 2: Ergänzungsband, Ed. Guy Van Kerckhoven, Husserliana Do- kumente II, vol. 2, Dordrecht, Kluwer, 1988, p. 259. Cf. Hua Mat VIII, 446.

55 Hua XV, 91.

56 Hua XV, 342. Cf. Hua XV, 74, 369.

57 Hua XV, 342. Cf. Hua Mat VIII, 198.

58 Hua XV, xlviii. Cf. Hua Mat VIII, 57.

59 Ms. E III 8, 9a. Cf. Hua XV, 370. Cf. Hua Mat VIII, 394.

60 Ms. A V 20, $9 b$.

61 Hua XV, 366.

62 Hua Mat VIII, 437. Cf. Hua XIII, 457. Cf. Hua XV, 366, 367.

63 Cf. Hua XIV, 244. Cf. Depraz, Natalie, Transcendence et incarnation. Le statut de l'intersubjectivité comme altérité à soi chez Husserl, París, Vrin, 1995, p. 259.
64 Cf. Kühn, Rolf, "Intersubjektivität und Gemeinschaftlichkeit", en Recherches Husserliennes, Centre de Recherches Phénoménologiques, vol. 21, 2004, p. 77.

65 Cf. Hua XV, 287.

66 Cf. Waldenfels, Bernhard, Grundmotive einer Phänomenologie des Fremden, Frankfurt a. Main, Suhrkamp, 2006, pp. 117-118.

67 Cf. Waldenfels, Bernhard, Das Zwischenreich des Dialogs. Sozialphilosophische Untersuchungen in Anschluss an Edmund Husserl, Phaenomenologica 41, Den Haag, Martinus Nijhoff, 1971, pp. 309, 322. 